\title{
CONCEPTUALIZATION AND CHARACTERIZATION OF INTRAPRENEURSHIP: AN EXPLORATORY STUDY ${ }^{i}$
}

\author{
CONCEPTUALIZACIÓN Y CARACTERIZACIÓN DEL INTRAEMPRENDIMIENTO: UN ESTUDIO \\ EXPLORATORIO
}

\section{CONCEPTUALIZAÇÃO E CARACTERIZAÇÃO DO INTRAEMPREGO: ESTUDO EXPLORATÓRIO}

\section{CITATION}

Galván-Vela, Esthela \& Sánchez-Limón, Mónica L. (2018) Conceptualization and characterization of intrapreneurship: an exploratory study. Dimensión Empresarial, 161-176. DOI: http://dx.doi.org/10.15665/dem.v16i2.1538

JEL: M3, M39

\begin{abstract}
This article corresponds to a qualitative type of research whose purpose is to represent the phenomenon of intrapreneurship in terms of its conceptualization and characterization through documentary analysis, as well as the application, transcription, coding and analysis of 11 interviews with people related with the corporate entrepreneurship in the northeast area of Mexico. The interactions with the research subjects led to the categorization of codes of first and second order in terms of connotation attributed by the respondent to the term (CO) and the characteristics of normative perception in terms of resources and capacities of intrapreneurial companies (CA). The subcodes attributed to such families allowed a contrast between the information derived from the contributions of the research subjects and the existing theoretical-empirical knowledge.
\end{abstract}

Key words: intrapreneurship, conceptualization, characterization, qualitative analysis.

\section{RESUMEN}

El presente artículo corresponde a un tipo cualitativo de investigación cuya finalidad es representar el fenómeno del intraemprendimiento en cuanto a su conceptualización y caracterización por medio del análisis documental, así como la aplicación, transcripción, codificación y análisis de 11 entrevistas realizadas a personas relacionadas con el emprendimiento en la empresa en la zona noreste de México. Las interacciones con los sujetos de investigación derivaron en la categorización de códigos de primer y segundo orden en cuanto a connotación atribuida por el entrevistado al término (CO) y las características de percepción normativa en términos de recursos y capacidades de las empresas intraemprendedoras (CA). Los subcódigos atribuidos a tales familias permitieron un contraste entre la información derivada de las aportaciones de los sujetos de investigación y el conocimiento teórico-empírico existente.

Palabras clave: intraemprendimiento, conceptualización, caracterización, análisis cualitativo.

\section{RESUMO}

Este artigo corresponde a um tipo qualitativo de pesquisa cujo objetivo é representar o fenômeno do intraemprego em termos de sua conceituação e caracterização por meio de análise documental, bem como a aplicação, transcrição, codificação e análise de 11 entrevistas feitas a pessoas relacionadas ao intraempreendedor na zona nordeste do México. As interações com os sujeitos da pesquisa levaram à categorização de códigos de primeira e segunda ordem em termos de conotação atribuídos pelo respondente ao termo $(\mathrm{CO})$ e as característica da percepção normativa em termos de recursos e capacidades de empresas intraempreoristas (CA). Os subcódigos atribuídos a essas famílias permitiram um contraste entre as informações derivadas das contribuições dos sujeitos da pesquisa e do conhecimento teórico-empírico existente. Palavras chave: intraemprego, conceituação, caracterização, análise qualitativa. 


\section{INTRODUCCIÓN}

El entorno actual en términos de competitividad empresarial es hoy, más que nunca, el principal aliciente para la procuración de la supervivencia y el desarrollo de los entes económicos (Baruah \& Ward, 2014; Morris, Kurakto \& Covin, 2000). La dinamicidad del contexto económico, político y social actual supone un ambiente de cambios continuos y acelerados en el que las empresas se han visto obligadas a adaptarse, por medio de innovaciones $\mathrm{O}$ transformaciones estructurales $\mathrm{O}$ estratégicas, a fin de asegurar su crecimiento y/o su posición competitiva (Aragón-Correa \& Sharma, 2003).

Ante tal panorama, se reconoce que el emprendimiento corporativo, resulta una forma efectiva de lograr la subsistencia y el desarrollo (Kuratko, 2010). Algunos autores argumentan que el mismo es un proceso clave en aspectos de competitividad o desarrollo macroeconómico (e.g. Parker, 2011; Turró, Urbano \& Peris-Ortiz, 2014) y otros, que representa un efecto revitalizante a nivel corporativo en cuanto a crecimiento relativo o absoluto (e.g. Antoncic \& Hisrich, 2001; Kearney, Hisrich \& Roche, 2010; Kearney, Hisrich \& Antoncic, 2013; Zahra, 1991); rendimiento o desempeño financiero (e.g. Covin \& Slevin, 1990; Kaerney et al., 2013); capacidad competitiva y reposicionamiento estratégico (e.g. Ireland, Covin y Kuratko, 2009); y, resultados de trabajo, como satisfacción laboral y compromiso afectivo (e.g. Rutherford y Holt, 2007).

Lo anterior fundamenta el interés de los teóricos de las ciencias económico-administrativas en el fenómeno del intraemprendimiento durante poco más de cuatro décadas. Teniendo por consiguiente antecedentes en la materia que datan de los años 70'sii (Antoncic, 2007; Kearney et al., 2010; Kuratko, 2010; Sakhdari, 2016; Turró et al., 2013, 2014; Zahra, 1991), década en la que investigadores como Collins y Moore (1970) distinguieron formalmente los emprendimientos dentro y fuera de la firma nombrándoles “emprendimiento corporativo" y "emprendimiento independiente", respectivamente.

Actualmente el comportamiento emprendedor en la firma es nombrado desde diferentes términos, como "emprendimiento corporativo" (Collins \& Moore, 1970; Covin \& Slevin, 1991; Dess, Lumpkin \& Covin, 1997; Guth \& Ginsberg, 1990; Kuratko \& Audretsch, 2013; Zahra, 1991); "emprendimiento corporativo interno" (Ellis \& Taylor, 1987; Schollhammer, 1981; Jones \& Butler, 1992); "emprendimiento estratégico" (Ketchen, Ireland \& Snow, 2007); “emprendimiento organizacional” (Kearney, Hisrich \& Antoncic, 2013); "intensidad emprendedora" (De Villiers-Scheepers, 2012); e "intraemprendimiento" (Pinchot, 1985).

Independientemente del término que se atribuya al comportamiento emprendedor en la empresa, ha quedado en claro que los dominios del mismo no sólo están restringidos a la creación de un nuevo negocio o producto (Covin \& Slevin, 1990), sino que involucra un conjunto de actos que reflejan una conducta de innovación, auto-renovación y/o proactividad, siendo estos atributos las dimensiones operacionales reconocidas en la literatura (e.g. Alpkan, Bulut, Gunday, Ulusoy \& Kilic, 2010; Altman \& Zacharakis, 2003; Antoncic, 2007; Baruah \& Ward, 2014; De VilliersScheepers, 2012; Guth \& Ginsberg, 1990; Hornsby, Kuratko \& Zahra, 2002; Ireland, Covin \& Kuratko, 2009; Kearney et al., 2013; Kuratko, 2010; Kuratko \& Audretsch, 2013; Kuratko, Hornsby \& Covin, 2014; Miller, 1983; Parker, 2011; Schindehutte, Morris \& Kuratko, 2000; Turró et al., 2014; Zahra, 1991).

Adicionalmente, el emprendimiento en la empresa incluye todo el conjunto de comportamientos orientados a la búsqueda y aprovechamiento de las oportunidades en el entorno o generación de ventajas para la creación de valor (Kearney et al., 2013; Kuratko et al., 2014), equilibrando de forma ambidiestra la exploración ambiental y la explotación estratégica de los recursos disponibles (De Villiers-Sheepers, 2012). En este sentido, la conducta intraemprendedora representa un conjunto de atributos necesarios e indispensables en el 
entorno competitivo actual (Kearney et al., 2013; Turró et al., 2014), sobre todo para empresas que se ven inmersas en la dinámica que compete a las economías emergentes (De Viellers-Sheepers, 2012; Weeks, 2008).

No obstante, a pesar de la existencia de aportaciones teóricas en la materia, aún son pocos los estudios que aportan nociones basadas en evidencia empírica (Kurakto \& Audrech, 2013) y, casi nulos aquellos que reúnen las aportaciones de sujetos inmersos en la dinámica comercial. Existen, por consiguiente, grandes desafíos en cuanto a la comprensión profunda del intraemprendimiento (Antoncic, 2007; Antoncic \& Hisrich, 2001; Hornsby et al., 2002; Schindehutte et al., 2000). La gran mayoría de las investigaciones, han centrado su atención en la relación entre el intraemprendimiento y el rendimiento en la empresa (Kearney et al., 2013), olvidando dejar en claro las percepciones y comportamiento de los líderes organizacionales con respecto al fenómeno.

En este estudio se reconoce la necesidad de subrogar las capacidades incipientes de las empresas en la actualidad por medio de la exploración profunda del emprendimiento corporativo (Parker, 2011). Adicionalmente, se considera la necesidad de incorporar nuevos métodos creativos para el contraste de la información disponible, tal cual aconsejan Zahra, Jennings y Kuratko (1999), en cuanto a que la existencia de modelos en la materia no garantiza la noción completa del fenómeno, sino que se requiere la exploración creativa.

Por lo anterior, en el presente estudio tiene como finalidad representar el fenómeno del intraemprendimiento en cuanto a su conceptualización y caracterización por medio del contraste entre la información resultante de aportaciones documentales previas, es decir, el conocimiento abstracto en la materia, y las nociones y percepciones reales de sujetos inmersos en el fenómeno, para con ello, dar respuesta a algunas interrogantes, como lo son: ¿cuál es el significado atribuido por los líderes organizacionales al intraemprendimiento? y ¿qué características consideran que debe tener una empresa intraemprendedora?

\section{METODOLOGÍA}

La presente investigación fue desarrollada acorde a técnicas cualitativas de investigación a fin de identificar la conceptualización y caracterización del fenómeno del intraemprendimiento. Para tal objeto, se siguieron métodos deductivos e inductivos, en los que, se partió, en una primera instancia, de la particularización de hechos documentados que corresponden al conocimiento abstracto o estado del arte del fenómeno; $y$, en una segunda instancia a la comparación de tales aportaciones con respecto a las opiniones, descripciones, creencias y opiniones de sujetos de investigación.

Como hemos señalado con anterioridad, se reconoce la necesidad de seguir contribuyendo al conjunto de nociones teórico-empíricas del emprendimiento en la empresa, debido a la carencia literaria con respecto al mismo en el contexto de las economías emergentes. En este sentido, la ambigüedad de la terminología atribuida al intraemprendimiento, así como la escasa literatura en la materia, han permitido definir a la presente en un alcance exploratorio.

Con la finalidad de dar respuesta a las interrogantes planteadas en el apartado anterior, se ha recurrido en un primer ejercicio a la clasificación documental de literatura, basándonos en la búsqueda de los principales términos con que se reconoce al emprendimiento en la empresa por medio de las herramientas que proporciona la Thompson Routers, específicamente la compilación principal de Web of Science ${ }^{\mathrm{TM}}$, en donde se lograron identificar 121 fuentes publicadas desde 1980 hasta 2017 para las categorías de administración, negocios y economía. Adicionalmente, una búsqueda en Scopus ${ }^{\circledR}$ bajo los mismos criterios de clasificación, permitió la compilación de 162 artículos. 
El procesamiento de estos documentos en el gestor bibliográfico Mandeley permitió la eliminación de duplicados, logrando así una colección de 227 fuentes, las cuales fueron procesadas mediante otras herramientas como Pajek, para el análisis de redes, y BibExcel, para su representación gráfica. Con lo anterior, se logró la identificación de autores con más publicaciones en la materia, sus redes de cooperación y otras cuestiones como palabras claves o variables relacionadas. Sin embargo, conocer los autores con más publicaciones no garantiza que los mismos se pudieran considerar los principales referentes en el estudio del intraemprendimiento. Para poder identificar a estos últimos, se realizó un contraste entre la información obtenida en el bibliométrico con las herramientas comunes de los buscadores de datos, como la visualización de aquellos con mayores índices de citación, para el caso de los documentos en WOS; y, la filtración basada en relevancia de documentos, para los contenidos de Scopus ${ }^{\circledR}$.

La compilación y análisis de algunos de los textos pioneros y más representativos en la materia, permitió la consolidación de una sólida base de información documental en lo que se refiere a la conceptualización y caracterización del intraemprendimiento. Lo anterior con la finalidad de enriquecer y contrastar los datos derivados de las aportaciones de nuestros sujetos de investigación, por medio del proceso de análisis de datos cualitativos propuesto por Miles y Huberman (1994) consistente en la recolección, reducción y organización de datos, así como la extracción y verificación de conclusiones.

Las contribuciones de los sujetos de investigación se lograron captar por medio de una segunda técnica de análisis que consistió en la aplicación de entrevistas focalizadas y estructuradas a individuos con características similares, es decir, emprendedores, empresarios y expertos en la materia, en su gran mayoría pertenecientes a la Confederación Patronal de la República Mexicana (COPARMEX) y a la Cámara
Nacional de Comercio (CANACO) en la zona norte de México.

Es importante hacer mención que se procuró en todo momento la realización de entrevistas bajo condiciones ambientales propicias, así como el seguimiento de un guion de sesión preestablecido a fin de captar la realidad desde los ojos de nuestros sujetos de investigación, por medio de sus opiniones, descripciones, creencias y experiencias en cuanto al fenómeno.

La elección de nuestra muestra fue intencionada, pues se preguntó a los líderes de los organismos mencionados, sobre aquellos individuos o empresas afiliadas más innovadoras o que hubiesen llevado a cabo algún emprendimiento. En este sentido se invitaron a 11 sujetos quienes en su totalidad accedieron a participar en el estudio, teniendo por consiguiente una tasa efectiva de respuesta del 100 por ciento.

Las entrevistas fueron realizadas de julio a noviembre de 2017. El tiempo promedio de las mismas fue de 1.2 horas. El procedimiento de aplicación consistió en una primera instancia en la presentación entrevistadorentrevistado y la exposición de las preguntas centrales de investigación. Posteriormente, la lectura de un acuerdo de confidencialidad, la exposición del objetivo del estudio, así como el planteamiento y respuesta de las preguntas del instrumento. Es necesario señalar que, a pesar de las nociones teóricas del entrevistado con respecto al fenómeno, se procuró en todo momento seguir criterios de no direccionalidad, especificidad, amplitud y congruencia durante la entrevista.

Toda la información derivada de las interacciones entrevistador-entrevistado fue grabada en audio bajo el consentimiento de los sujetos de investigación, para posteriormente ser transcritas y codificadas por medio de la clasificación de datos fragmentados en el software Atlas Ti7. Una vez transcritas las entrevistas, se procedió a la realización de un proceso de codificación provisional por categorías, bajo la metodología de Miles, Huberman y Saldaña (2013), la cual resulta pertinente para los estudios cualitativos que se basan, corroboran o amplían 
investigaciones previas. No obstante, en un segundo ejercicio se procedió a la reducción y clasificación de datos por medio de la codificación abierta de Strauss y Corbin (1990), haciendo uso de las representaciones in vivo, descriptiva y de evaluación.

Ambos ejercicios permitieron el establecimiento de categorías o grupos de códigos entre cada conjunto de datos, los cuales serían contrastados con la información resultante del análisis literario. Lo anterior permitió una comparativa entre las aportaciones de sujetos de investigación inmersos en la dinámica de negocios, es decir, el conocimiento real del fenómeno, con el contenido documental disponible en la materia, es decir, el conocimiento teórico o abstracto. El siguiente apartado expone los resultados derivados de los procedimientos anteriormente descritos.

\section{RESULTADOS}

En el presente se contemplan algunos aspectos generales con respecto a las características de los sujetos de investigación; la codificación de sus contribuciones; la categorización de los códigos de primer y segundo orden derivados de la información resultante de las entrevistas; y la validación de los códigos por medio del contraste entre los datos obtenidos en las entrevistas y la información teórico-empírica disponible en materia de intraemprendimiento.

En cuanto a los sujetos de investigación cabe señalar que los mismos son individuos cuyo rango se encuentra en la escala jerárquica principal para la toma de decisiones en sus respectivas empresas, es decir, propietarios y/o administradores generales. Así mismo, han contribuido expertos en cuestiones de innovación, emprendimiento individual y emprendimiento corporativo, específicamente líderes en programas en la materia en diferentes instituciones de educación superior, representando los mismos un 27 por ciento de la muestra total.
De los entrevistados se tiene también que un 64 por ciento son hombres y 36 por ciento son mujeres, cuya media de edad se sitúa en 41.8 años, donde el límite superior es 55 y el límite inferior es 24 . Se tiene también que las empresas que representan los individuos son en su mayoría de giro comercial como restaurantes, papelerías, madereras y tiendas de ropa (45 por ciento); así como empresas correspondientes a la clasificación de servicios de salud y cuidado personal (27 por ciento). Estas empresas poseen una plantilla laboral cuya media se sitúa en 26.62, cuyos límites superior e inferior son de 63 y 8 empleados, respectivamente. Es importante aclarar que la totalidad de las empresas se encuentran situadas en el noreste de México. Ahora bien, las respuestas emitidas por los entrevistados durante la aplicación de nuestro instrumento de investigación, así como las nociones conceptuales previas sobre el fenómeno, nos han permitido categorizar los códigos emitidos en diferentes familias (tabla 1), entre ellas: aquellas que corresponden al concepto atribuido por el entrevistado al intraemprendimiento (CO); las características de las empresas intraemprendedoras (CA); y las recomendaciones emitidas por los entrevistados (RE)

Es importante mencionar que, para cada una de las familias o categorías, se tienen un conjunto de códigos y subcódigos agrupados, puesto que cada una ha derivado en una serie notable de menciones a lo largo de la aplicación de nuestro instrumento. Estos mismos, han sido analizados a partir de diferentes procesos donde en un primer ejercicio, se ha compilado información documental disponible en diferentes repositorios de información académico-científica. En una segunda instancia, se han recopilado información acorde a experiencias, opiniones y creencias desde los ojos de nuestros sujetos de investigación y, por último, se ha llevado a cabo un contraste entre la información emitida por los individuos y la información documental disponible. Se exponen en los subapartados siguientes, cada uno de los resultados en las diversas categorías. 
Como hemos mencionado en el primer apartado, son diversas son las terminologías con que se ha reconocido a la conducta intraemprendedora (emprendimiento corporativo, emprendimiento corporativo interno, emprendimiento estratégico, emprendimiento organizacional, intensidad emprendedora e intraemprendimiento). A la par de esta terminología, múltiples son los autores que han buscado definir conceptualmente el fenómeno (Antoncic \& Hisrich, 2001; De Villiers-Scheepers, 2012; Kearney et al., 2013; Schindehutte et al., 2000; Turró et al., 2014; Zahra, 1991).

Tabla 1.

Categorización de familias de códigos

\begin{tabular}{|l|l|l|}
\hline \multicolumn{1}{|c|}{ CATEGORÍA } & CÓDIGO & \multicolumn{1}{c|}{ DESCRIPCIÓN } \\
\hline Conceptualización & CO & Término o connotación que atribuye el entrevistado al intraemprendimiento. \\
\hline Caracterización & CA & $\begin{array}{l}\text { Características de percepción normativa en términos de recursos y capacidades } \\
\text { de las empresas intraemprendedoras. }\end{array}$ \\
\hline Recomendaciones & RE & $\begin{array}{l}\text { Percepción de características faltantes en empresas mexicanas para el desarrollo } \\
\text { del comportamiento intraemprendedor. }\end{array}$ \\
\hline
\end{tabular}

Fuente: elaboración propia

Haciendo una breve retrospectiva en materia de intraemprendimiento encontramos orígenes conceptuales que datan de la década de los setenta, mediante la distinción expuesta por Collins y Moore (1970) entre emprendimientos independientes y emprendimientos corporativos, los cuales serían definidos más adelante por Sharma y Chrisman (1999), quienes expusieron al primero como “... el proceso mediante el cual un individuo o grupo de individuos, actuando independientemente de cualquier asociación con una organización existente, crea una nueva organización" (p.18); y, al segundo como: “... el proceso mediante el cual un individuo o un grupo de personas, en asociación con una organización existente, crea una nueva organización o instiga la renovación o la innovación dentro de esa organización" (p.18).

No obstante, algunas dimensiones relacionadas al mencionado comportamiento se remontan a investigaciones más arcaicas. Schumpeter (1934), por ejemplo, ha sido uno de los principales referentes en materia de innovación y emprendimiento, el autor, a agrandes rasgos consideró que la producción era el resultado de una amalgama de fuerzas materiales e inmateriales. Los primeros dados por los factores de producción común (trabajo, tierra y capital) y, los últimos, representados por los factores internos relacionados con la tecnología, la innovación y la cultura, siendo estos últimos más dinámicos y decisivos.

Entre otras aportaciones, la Teoría del Desenvolvimiento Económico de Schumpeter (1934) también nos introdujo a aspectos relacionados al comportamiento intraemprendedor en las firmas, al sustentar el fenómeno como consecuencia de cambios exógenos de las organizaciones, en el que, la dinámica del desarrollo externo y las combinaciones de aprendizaje existente e incremental en la empresa, son catalizadores de innovaciones radicales, tales como la introducción de nuevos productos o servicios, la apertura de nuevos mercados, el desarrollo de nuevos medios de producción y logística, o cambios revolucionarios en la estructura de la organización, o en sus métodos de gestión o procesos; y, generación de nuevas fuentes de materias primas.

No obstante, se sabe que las ideas de Schumpeter fueron olvidadas en la literatura sobre gestión de la empresa 
durante algunas décadas posteriores a su desarrollo (Yoguel, Barletta \& Pereira, 2013), empero, gracias a autores como Nelson \& Winter $(1982,2002)$ y Teece \& Pisano (1994) y colaboradores $(1997,2013)$, es que surge un pensamiento neoschumpeteriano en torno a la innovación y las capacidades dinámicas. Esta visión es, por ende, la fundamentación teórica en el campo del emprendimiento empresarial o intraemprendimiento, exponiéndose en gran cantidad de textos al mencionado autor como el precedente de tales teorías, y el referente histórico del estudio del fenómeno del emprendimiento en la empresa (e. g. Antoncic, 2007; Guth \& Ginsberg, 1990; Miller, 1983; Turró et al., 2014; Zahra, 1991,1993).

Adicionalmente, hoy se atribuyen al emprendimiento corporativo, algunas nociones que demuestran la complejidad del fenómeno. Por un lado, Lumpkin y Dess (1996), mencionan que el mismo se presenta a nivel individual, grupal y organizacional. Por otro, Stevenson y Jarrillo (1990) enuncian que el fenómeno ha sido nutrido en torno a diferentes disciplinas, como lo son: la sociología y psicología, las cuales proveen un espacio de reflexión en cuanto al individuo y su importancia, así como las variables de su entorno; la administración, que estudia las prácticas de gestión y el comportamiento estratégico y organizacional; y la economía, al atribuir al intraemprendimiento factores consecuentes como el crecimiento y desarrollo.

Con base en lo anterior, el emprendimiento corporativo sigue siendo un tópico de interés para diferentes disciplinas, sobre todo, en el campo de la gestión y la estrategia de negocios. Esto fundamenta las aportaciones de los estudiosos en la materia, quienes han realizado diversas aportaciones que permiten entender su significado, sus características, sus factores de origen y sus consecuencias. Sin embargo, al igual que sucede con muchas de las definiciones científicas en materia de emprendimiento, se carece de una definición única y consensuada al término (Coduras, Guerrero \& Peña, 2011, Hornsby et al., 2002; Lumpking \& Dess, 1996; Morris \& Kuratko, 2002; Parker, 2011).

La tabla 2 resume algunas de las contribuciones más significativas para el intraemprendimiento. Donde, según su evolución, las primeras definiciones relacionaban el mismo con el desarrollo de unidades independientes a una organización. Hoy dicho proceso es reconocido como sólo una dimensión de la amalgama de actos relacionados al fenómeno llamada corporate venturingiii. Más adelante se incorporarían aspectos referentes a la diversificación, renovación o reestructuración estratégica y estructural en la empresa, consecuentes de la optimización eficiente de los recursos disponibles. Aspectos como la innovación, y las condiciones hostiles o dinamicidad y fluctuaciones en el ambiente externo de la empresa han tomado poco a poco relevancia.

Las condiciones del entorno de la empresa y su adecuada exploración y explotación también son consideradas como un incentivo para el nacimiento de nuevas empresas en organizaciones existentes que finalmente desemboca en cuestiones de rentabilidad y posicionamiento estratégico y competitivo. Otras cuestiones como cultura emprendedora no son adicionadas a las conceptualizaciones sino hasta las primeas aportaciones del siglo XXI, cuando se comienzan a explorar las condiciones de la filosofía empresarial como detonantes del cambio radical e incremental. 
Tabla 2.

Conceptualización del intraemprendimiento: aportaciones teóricas.

\begin{tabular}{|c|c|}
\hline AUTORES & CONCEPTO \\
\hline $\begin{array}{l}\text { Collins y Moore } \\
(1970)\end{array}$ & $\begin{array}{l}\text { Se distinguen dos tipos de emprendimientos: 1) emprendimiento corporativo; 2) emprendimiento } \\
\text { independiente. }\end{array}$ \\
\hline $\begin{array}{l}\text { Burgelman } \\
(1983)\end{array}$ & $\begin{array}{l}\text { El espíritu empresarial se refiere al proceso mediante el cual las empresas se dedican a la } \\
\text { diversificación a través del desarrollo informal. Dicha diversificación requiere nuevas combinaciones } \\
\text { de recursos para extender las actividades de la empresa en áreas no relacionadas o marginalmente } \\
\text { relacionadas con su actual dominio de competencia y el correspondiente conjunto de oportunidades } \\
\text { (p.1349). }\end{array}$ \\
\hline $\begin{array}{l}\text { Burgelman } \\
(1984)\end{array}$ & $\begin{array}{l}\text {...es extender el dominio de competencia y correspondiente conjunto de oportunidades a través de } \\
\text { la generación interna de nuevas combinaciones de recursos (p. 154). }\end{array}$ \\
\hline $\begin{array}{l}\text { Nielsen, Peters y } \\
\text { Hisrich (1985) }\end{array}$ & $\begin{array}{l}\text { Intraemprendimiento es el desarrollo dentro de una gran organización de mercados internos y } \\
\text { unidades relativamente pequeñas e independientes diseñadas para crear, internamente probar } \\
\text { mercado y expandir servicios mejorados o innovadores de personal, tecnologías o métodos dentro } \\
\text { de la organización. Esto difiere de las grandes unidades empresariales cuyo propósito es desarrollar } \\
\text { posiciones rentables en mercados externos (p. 181). }\end{array}$ \\
\hline Pinchot (1985) & $\begin{array}{l}\text { Es el desarrollo de mercados en el interior de una organización y relativamente de unidades } \\
\text { independientes diseñadas para crear, internamente, probar y ampliar mercados y/o servicios } \\
\text { innovadores, tecnológicos o métodos. }\end{array}$ \\
\hline Knight (1987) & $\begin{array}{l}\text { El emprendimiento es una postura fundamental, instrumentalmente importante para la innovación } \\
\text { estratégica, particularmente bajo condiciones cambiantes en el entorno externo de la empresa. La } \\
\text { noción de orientación empresarial es aplicable a cualquier empresa, independientemente de su } \\
\text { tamaño y tipo (p. 215) }\end{array}$ \\
\hline $\begin{array}{l}\text { Jennings y } \\
\text { Lumpkin (1989) }\end{array}$ & $\begin{array}{l}\text { Es un concepto multidimensional que incorpora las actividades de una empresa dirigidas a la } \\
\text { innovación de productos y tecnológica, a la toma de riesgos y la proactividad (p. 486). }\end{array}$ \\
\hline $\begin{array}{l}\text { Guth y } \\
\text { Ginsberg (1990) }\end{array}$ & $\begin{array}{l}\text { El emprendimiento empresarial abarca dos tipos de fenómenos y los procesos que los rodean: (1) el } \\
\text { nacimiento de nuevos negocios dentro de las organizaciones existentes, es decir, la innovación interna } \\
\text { o el riesgo; Y (2) la transformación de las organizaciones a través de la renovación de las ideas clave } \\
\text { sobre las que se construyen, es decir, la renovación estratégica (p.5). }\end{array}$ \\
\hline Zahra (1991) & $\begin{array}{l}\text { Se refiere al proceso de creación de nuevos negocios dentro de las empresas establecidas para mejorar } \\
\text { la rentabilidad de la organización y mejorar la posición competitiva de una empresa o la renovación } \\
\text { estratégica de negocio existente (pp. 260-261). }\end{array}$ \\
\hline $\begin{array}{l}\text { Covin y Slevin } \\
(1991)\end{array}$ & $\begin{array}{l}\text { El emprendimiento corporativo es descrito como una dimensión de la postura estratégica } \\
\text { representada por la propensión de la firma a la toma de riesgos, la tendencia a actuar agresivos contra } \\
\text { la competencia, el establecimiento de métodos proactivos y la innovación de productos (p. 7) }\end{array}$ \\
\hline Zahra (1993) & $\begin{array}{l}\text {... un proceso de renovación organizacional que tiene dos dimensiones distintas pero relacionadas: la } \\
\text { innovación y riesgo [venturing], y la renovación estratégica (p. 321) }\end{array}$ \\
\hline $\begin{array}{l}\text { Lumpkin y Dess } \\
(1996)\end{array}$ & $\begin{array}{l}\text { El acto esencial del emprendimiento es la nueva entrada. Se puede lograr una nueva entrada } \\
\text { ingresando a mercados nuevos o establecidos con bienes o servicios nuevos o existentes. La nueva } \\
\text { entrada es el acto de lanzar una nueva empresa, ya sea por una empresa nueva, a través de una } \\
\text { empresa existente o por medio de una empresa corporativa interna... El EO [orientación } \\
\text { emprendedora] se refiere a los procesos, prácticas y actividades de toma de decisiones que conducen } \\
\text { a una nueva entrada (p. 136-137) }\end{array}$ \\
\hline $\begin{array}{l}\text { Sharma y } \\
\text { Chrisman (1999) }\end{array}$ & $\begin{array}{l}\text {... El proceso mediante el cual un individuo o un grupo de individuos, en asociación con una } \\
\text { organización existente, crea una nueva organización o instiga la renovación o la innovación dentro } \\
\text { de esa organización " (p.18) }\end{array}$ \\
\hline $\begin{array}{l}\text { Antonic y } \\
\text { Hisrich (2001) }\end{array}$ & $\begin{array}{l}\text { Se refiere al proceso que ocurre en el interior de una firma independientemente de su tamaño y que } \\
\text { no sólo conduce a nuevos negocios, sino también a otras actividades innovadoras como el desarrollo } \\
\text { de productos, servicios, tecnologías, técnicas administrativas, estrategias o posturas competitivas } \\
\text { (p.498). }\end{array}$ \\
\hline
\end{tabular}




\begin{tabular}{|c|c|}
\hline AUTORES & CONCEPTO \\
\hline $\begin{array}{l}\text { Antonic y } \\
\text { Hisrich }(2003)\end{array}$ & $\begin{array}{l}\text { Se define como un emprendimiento dentro de una organización existente, refiriéndose a las } \\
\text { intenciones conductuales emergentes y comportamientos organizacionales relacionados con las } \\
\text { desviaciones de lo acostumbrado. Los procesos intraemprendedores continúan dentro de una } \\
\text { empresa existente, independientemente de su tamaño (p. 9). }\end{array}$ \\
\hline $\begin{array}{l}\text { Kuratko, } \\
\text { Ireland, Covin y } \\
\text { Hornsby (2005) }\end{array}$ & $\begin{array}{l}\text { Comportamiento que requiere sanciones organizacionales y compromisos de recursos con el } \\
\text { propósito de desarrollar diferentes tipos de innovaciones creadoras de valor (p.700). }\end{array}$ \\
\hline $\begin{array}{l}\text { Morris y } \\
\text { Kuratko (2006) }\end{array}$ & $\begin{array}{l}\text { Es un término utilizado para describir el comportamiento emprendedor que puede desarrollarse en } \\
\text { el seno de medianas y grandes organizaciones establecidas, que incluye términos como } \\
\text { organizaciones emprendedoras, intraemprendimiento y capital riesgo. }\end{array}$ \\
\hline $\begin{array}{l}\text { Rutherford y } \\
\text { Holt (2007) }\end{array}$ & $\begin{array}{l}\text { EC es el proceso de mejorar la capacidad de la firma para adquirir y utilizar las destrezas y habilidades } \\
\text { innovadoras de los miembros de la empresa (p. 430). }\end{array}$ \\
\hline $\begin{array}{l}\text { Ireland, Covin y } \\
\text { Kurakto (2009) }\end{array}$ & $\begin{array}{l}\text { Una estrategia de emprendimiento corporativo es una visión directa o visión dirigida en el } \\
\text { comportamiento emprendedor que a propósito y continuamente rejuvenece a la organización y da } \\
\text { forma al alcance de sus operaciones a través del reconocimiento y la explotación de oportunidades } \\
\text { empresariales (p. 21) }\end{array}$ \\
\hline Parker (2011) & $\begin{array}{l}\text { Es una práctica para el desarrollo de nuevas empresas dentro de una organización existente, para } \\
\text { explotar una nueva oportunidad y crear un valor económico. }\end{array}$ \\
\hline $\begin{array}{l}\text { De Villiers- } \\
\text { Sheepers (2012) }\end{array}$ & $\begin{array}{l}\text { Emprendimiento corporativo es un fenómeno multidimensional. Describe el proceso mediante el } \\
\text { cual las firmas establecidas pueden actuar innovativa, arriesgada y proactivamente. Las empresas de } \\
\text { riesgo, el intraemprendimiento y la renovación estratégica son componentes diferentes del } \\
\text { emprendimiento corporativo. }\end{array}$ \\
\hline $\begin{array}{l}\text { Kearney, } \\
\text { Hisrich y } \\
\text { Antonic (2013) }\end{array}$ & $\begin{array}{l}\text { Un conjunto de actividades a nivel de organización que se centran en el descubrimiento y la búsqueda } \\
\text { de nuevas oportunidades a través de actos de empresas de riesgo, renovación, innovación, la asunción } \\
\text { de riesgos y la proactividad (pp. 328-329). }\end{array}$ \\
\hline $\begin{array}{l}\text { Ahmed y Amjad } \\
\text { (2013) }\end{array}$ & $\begin{array}{l}\text { Intraemprendimiento es el acto de comportarse como un emprendedor dentro de los entornos } \\
\text { corporativos (p.88). }\end{array}$ \\
\hline
\end{tabular}

En relación con lo anterior, notoriamente la mayoría de los autores, independiente de la temporalidad de sus aportaciones, se refieren al emprendimiento corporativo como un proceso (e. g. Antoncic \& Hisrich, 2001, 2003; Burgelman, 1983; De Villiers-Sheepers, 2012; Rutherford \& Holt, 2007; Sharma \& Chrisman, 1999; Zahra, 1991, 1993). Otros tantos le distinguen también como una serie de actividades o acciones de la organización (e. g. Antoncic \& Hisrich, 2001, Burgelman, 1984; Jennings \& Lumpkin, 1999; Kearney et al., 2013; Lumpkin y Dess, 1996; Pinchot, 1985). Algunos como una serie de prácticas (Parker, 2011); o como una capacidad (Rutherford \& Holt, 2007); y otras tantas aportaciones más recientes le describen en relación a cuestiones del comportamiento, en lo que se refiere a la postura de la empresa (Knight, 1997); una visión dirigida (Ireland et al., 2009); una intención conductual (Antoncic \& Hisrich, 2003) o el comportamiento organizacional en general (Kuratko et al., 2005; Morris \& Kuratko, 2006; Ireland et al., 2009; Ahmed \& Amjad, 2013).

Entre otras cuestiones fundamentadas en la literatura y atribuidas al término del intraemprendimiento se tiene que la gran mayoría de las conceptualizaciones aluden a la dimensión de la innovación, sea la misma con relación a nuevos o sustancialmente mejorados productos, servicios, métodos de organización internos, estrategias y/o tecnologías (Covin \& Slevin, 1991; Jennings \& Lumpkin, 1999; Knight, 1997; Kearney et al., 2013; Kuratko et al., 2005; Sharma \& Chrisman, 1999; Zahra, 1993). Esta innovación estaría dada debido a la exploración y explotación de nuevos mercados (Nielsen 
et al., 1985; Pinchot, 1985; Lumpkin \& Dess, 1996) y el aprovechamiento de oportunidades en el entorno (Burgelman, 1984; Ireland et al., 2009; Parker, 2011).

Además de la innovación, la cual podría considerarse la dimensión más fundamentada, los autores generalmente enuncian en sus contribuciones conceptuales algunas otras dimensiones representativas del fenómeno como lo son la apertura de nuevos negocios, la renovación estratégica, la proactividad y la asunción o toma de riesgos (Antoncic \& Hisrich, 2001; Covin \& Slevin, 1991; De Villiers-Sheepers, 2012; Guth \& Gingsberg, 1990; Jennings \& Lumpkin, 1999; Kearney et al., 2013; Ireland et al, 2009; Sharma \& Chrisman, 1999; Zahra, 1991, 1993).

Así mismo, entre las capacidades de la empresa se sustentan la adecuada combinación de los recursos (Burgelman, 1983, 1984; Kuratko et al., 2005; Ireland et al., 2009). Y, entre otros aspectos alusivos a los resultados del comportamiento emprendedor en la organización, se tienen la creación de valor (Kuratko et al., 2005; Parker, 2011), la mejora de la habilidad innovadora (Rutherford \& Holt, 2007); la mejora de la posición competitiva (Zahra, 1991) y la extensión del dominio de la competencia (Burgelman, 1983, 1984; Covin \& Slevin, 1991).

Además de las contribuciones de los teóricos en cuanto a la conceptualización, se han codificado y analizado la información resultante de las interacciones con los sujetos de investigación. La tabla 3 resume la codificación otorgada para sus contribuciones. En donde en un primer acercamiento los sujetos de investigación presentan un desconocimiento por la palabra "intraemprendimiento", no obstante, tras escuchar el término, éste es relacionado con el emprendimiento surgido dentro de la empresa (8 menciones [m]) y, contrariamente a la interpretación de los teóricos al describir el fenómeno como un proceso, los sujetos de investigación enuncian al mismo como un fenómeno interno $(7 \mathrm{~m})$; un tipo de capacidad $(1 \mathrm{~m})$ o una práctica interna $(1 \mathrm{~m})$. Adicionalmente, tal como se presenta en la literatura, la información desde la voz de los sujetos ha figurado al emprendimiento corporativo con aspectos de innovación en general $(6 \mathrm{~m})$ o de nuevos productos $(4 \mathrm{~m})$, empresas $(2 \mathrm{~m})$, servicios $(2 \mathrm{~m})$, procesos $(2 \mathrm{~m})$, técnicas (1m) y mercados (1m).

Tabla 3.

Conceptualización de intraemprendimiento: aportaciones en entrevistas.

\begin{tabular}{|l|c|}
\hline \multicolumn{1}{|c|}{ CÓDIGO } & FUNDAMENTADO \\
\hline CO: Desconocimiento del término & 8 \\
\hline CO: Emprendimiento en la empresa & 8 \\
\hline CO: Fenómeno interno & 7 \\
\hline CO: Innovación & 6 \\
\hline CO: Solución a problemas & 5 \\
\hline CO: Nuevos productos & 4 \\
\hline CO: Oportunidades en el ambiente & 2 \\
\hline CO: Cambio interno & 2 \\
\hline CO: Nuevas empresas & 2 \\
\hline CO: Nuevos servicios & 2 \\
\hline CO: Nuevos procesos & 2 \\
\hline CO: Nuevas técnicas & 1 \\
\hline CO: Nuevos mercados & 1 \\
\hline CO: Tipo de capacidad & 1 \\
\hline CO: Práctica interna & 1 \\
\hline
\end{tabular}

Fuente: elaboración propia a partir de datos en Atlas Ti7. 
Así mismo, se ha atribuido al emprendimiento corporativo la capacidad de la solución de problemas reales $(5 \mathrm{~m})$ o la atención de las oportunidades externas o ambientales (2m). Se argumenta, por ejemplo: “...Bueno mi concepción personal del intraemprendimiento es el fenómeno o las prácticas que surgen hacia el interior de una organización, y que fomenta el desarrollo de soluciones para atender oportunidades en el mercado o problemas que existen el mercado" (2: 2).

Es importante aclarar que entendemos ambos códigos (solución de problemas y atención de oportunidades) como aspectos relacionados, puesto que el desarrollo de soluciones es una consecuencia a la demanda del mercado, satisfacción de una necesidad o el aprovechamiento de una oportunidad latente; es decir “...la empresa emprendedora no está [del todo] preparada; la empresa emprendedora no está esperando la orden del superior para desarrollar una solución, [sino que] desarrolla la solución porque vio la oportunidad de mercado" (3:55).

Por otro lado, al emprendimiento corporativo, también se le ha relacionado con los cambios. Se ha mencionado, por ejemplo: “... intraemprendimiento es la serie de cambios que puedes hacer para crecer o mejorar. Tienes que emprender para cambiar y cambiar para emprender. Ese es el dilema, así como el del buevo y la gallina..." (5: 5); Así mismo se específica: “...son todos los cambios internos que hago en mi negocio, siempre que sean significativos" (6:2). El cambio también ha sido referido al intraemprendimiento como su característica más fundamentada.

Es importante en este punto señalar que, con el objeto de nutrir el concepto en base a las aportaciones de las percepciones y conocimiento real de los sujetos de investigación, se ha reunido también información referente a la caracterización que los mismos otorgan a las empresas que desarrollan este tipo de comportamiento. En este sentido, la tabla 4 resume la codificación y fundamentación de tales características, donde, notoriamente se tiene que la apertura al cambio resulta una de las variables con más fundamentación (11 menciones [m]), ante tal aspecto, los individuos manifiestan cuestiones como la conducta relacionada a la cultura y filosofía organizacional en pro del emprendimiento en general; la visión estratégica orientada al cambio; la postura individual de los trabajadores y el soporte de la administración.

Tabla 4.

Caracterización de intraemprendimiento: aportaciones en entrevistas.

\begin{tabular}{|l|c|}
\hline \multicolumn{1}{|c|}{ CÓDIGO } & FUNDAMENTADO \\
\hline CA: Apertura al cambio & 11 \\
\hline CA: Recurso humano: proactivo & 6 \\
\hline CA: No tradicionalista & 5 \\
\hline CA: Conciencia colectiva & 4 \\
\hline CA: Liderazgo efectivo & 3 \\
\hline CA: Innovación & 3 \\
\hline CA: Automotivación & 3 \\
\hline CA: Recurso humano: capacitado & 3 \\
\hline CA: Visión emprendedora & 3 \\
\hline CA: Desarrollo de soluciones & 2 \\
\hline CA: Comunicación efectiva & 2 \\
\hline CA: Proactividad & 2 \\
\hline CA: Búsqueda de crecimiento & 2 \\
\hline
\end{tabular}




\begin{tabular}{|l|c|}
\hline \multicolumn{1}{|c|}{ CÓDIGO } & FUNDAMENTADO \\
\hline CA: Persistencia individual & 2 \\
\hline CA: Recurso humano: innovador & 1 \\
\hline
\end{tabular}

Fuente: elaboración propia a partir de datos en Atlas Tỉ.

Otro aspecto que considerar como una característica importante de las empresas intraemprendedoras, es aquella que tiene que ver con las cualidades, aptitudes habilidades y destrezas del recurso humano. Se menciona, por ejemplo, que las empresas emprendedoras poseen una plantilla laboral proactiva $(6 \mathrm{~m})$, capacitada $(3 \mathrm{~m})$ e innovadora $(3 \mathrm{~m})$, así mismo, la conciencia colectiva de la empresa debería estar soportada en base al trabajo en equipo y el soporte a las ideas de los compañeros de trabajo y la alta gerencia $(4 \mathrm{~m})$. En tanto a aspectos relacionados a las capacidades individuales se manifiesta la importancia de la automotivación $(4 \mathrm{~m})$ y la persistencia individual $(2 \mathrm{~m})$, ambas cuestiones, también relacionadas con la actitud de la alta gerencia y los trabajadores.

Adicionalmente, entre algunas de las capacidades gerenciales de las empresas, se argumenta el liderazgo efectivo (3m), entendiendo a mismo como aquél capaz de vislumbrar, organizar e implementar nuevos proyectos que conlleven a resultados; la comunicación efectiva (2m), es decir, la capacidad de trasmitir ideas sin dar lugar a las interpretaciones erróneas entre la plantilla laboral; y el desarrollo de soluciones (2m) que, entre otras cosas, conlleven al desarrollo organizacional y al crecimiento de la organización (2m). Entre otras características generales, los entrevistados hacen alusión a dimensiones del intraemprendimiento las cuales han sido debatidas previamente, como lo son: la innovación $(3 \mathrm{~m})$, la proactividad $(2 \mathrm{~m})$, la visión emprendedora (3m), y las actitudes no tradicionalistas, todas ellas desde una perspectiva individual y organizacional.

\section{CONCLUSIONES}

El intraemprendimiento juega un papel protagónico en el crecimiento y desarrollo de las empresas en la actualidad. No obstante, aún resultan escasas las aportaciones teórico y/o empíricas que permitan un entendimiento más profundo de tal comportamiento, sobre todo aquellas derivadas de la voz de quienes se encuentran inmersos en la dinámica comercial día con día.

En el presente se reconoció la necesidad de la exploración del emprendimiento corporativo, así como la adición de nuevas formas de contrastar la información sobre el fenómeno. Por ello, la finalidad de esta investigación consistió en la representación del intraemprendimiento en cuanto a su conceptualización y caracterización por medio de un contraste entre información abstracta e información real en torno al mismo.

En este sentido se plantearon dos preguntas de investigación: ¿cuál es el significado atribuido por los líderes organizacionales al intraemprendimiento? y ¿qué características consideran que debe tener una empresa intraemprendedora? Para dar respuesta a las preguntas enunciadas, se realizó, en primera instancia una búsqueda documental profunda en bases de datos. Después a la aplicación de entrevistas de investigación a actores sociales relacionados ampliamente con el emprendimiento individual, corporativo y/o innovación; 
y, por último, a la transcripción, codificación, análisis y contraste de los datos.

Además de los resultados anteriormente presentados, en donde se expone la información alusiva a las representaciones conceptuales del fenómeno tanto por teóricos como por sujetos de investigación, y, como respuesta a las preguntas de investigación planteadas, hemos llegado a la siguiente noción conceptual con respecto al emprendimiento corporativo:

El intraemprendimiento representa la práctica o comportamiento emprendedor en las empresas, independientemente de su giro o tamaño, caracterizado por una filosofía de apertura al cambio, así como una visión general dirigida a la exploración y/o explotación de las oportunidades del entorno por medio de una adecuada combinación de recursos y el desarrollo de actividades proactivas e innovadoras, como la creación y/o mejora sustancial de productos, servicios, métodos de organización internos, procesos, tecnologías y mercados. Como proceso, la práctica intraemprendedora conlleva al desarrollo general de la empresa, o a aspectos específicos, como la mejora en el desempeño financiero, la capacidad competitiva, el reposicionamiento estratégico, el crecimiento relativo y absoluto y/o la satisfacción de los involucrados.

Lo anterior involucra el reconocimiento del intraemprendimiento más allá de una intención conductual, sino como un conjunto de actividades o comportamiento en el que las condiciones exógenas y endógenas de la empresa permiten la explotación de sus recursos y sus capacidades en el desarrollo de innovaciones en todas sus formas contribuyendo de esta forma a alguna mejora en la empresa.

Se considera que el contenido en el presente contribuye al esclarecimiento del término dadas las actuales ambigüedades conceptuales que se presentan en la creciente literatura en materia de emprendimiento corporativo. En cuanto a sus aportaciones prácticas, nos hemos dado a la tarea de definir y caracterizar un comportamiento, así también aludir algunas de las ventajas que supone el emprendimiento corporativo, como un acercamiento a la orientación implícita que las empresas interesadas en la comprensión conceptual del intraemprendimiento como un precedente para el establecimiento de nuevas y mejores prácticas en la empresa.

\section{REFERENCIAS}

Ahmed, S. N. \& Anjad, M. (2013). Job Satisfaction and Intrapreneurship: The moderating effect of Personality Trait. Journal of International Studies, 6 (1), 87-95

Altman, J. W. \& Zacharakis, A. (2003). An Integrative Model for Corporate Venturing. The Journal of Private Equity, 6 (4), 68-76. doi: https://doi.org/10.3905/jpe.2003.320057

Alpkan, L., Bulut, C., Gunday, G., Ulusoy, G. \& Kilic, K. (2010). Organizational support for intrapreneurship and its interaction with human capital to enhance innovative performance. Management Decision, 48 (5), 732-755. doi: http://dx.doi.org/10.1108/00251741011043902

Antoncic, B. (2007). Intrapreneurship: A comparative structural equation modeling study. Industrial Management and Data Systems, 107 (3), 309-325. doi: https://doi.org/10.1108/02635570710734244

Antoncic, B. \& Hisrich, R. D. (2001). Intrapreneurship: Construct refinement and crosscultural validation. Journal of Business Venturing, 16 (5), 495-527. doi: https://doi.org/10.1016/S0883-9026(99)00054-3

Aragón-Correa, J. A. \& Sharma, S. (2003). A contingent resource based view of proactive corporate environmental strategy. Academy of Management Review, 28 (1), 71-88 
Baruah, B. \& Ward, A. (2014). Metamorphosis of intrapreneurship as an effective organizational strategy. International Entrepreneurship and Management Journal, 11 (4), 811-822. doi: https://doi.org/10.1007/s11365-014-0318-3

Burgelman, R. A. (1983). A process model of internal corporate venturing in the diversified major firms. Administrative Science Quarterly, 28 (2), 223-244. doi: https://doi.org/10.2307/2392619

Burgelman, R. A. (1984). Designs for corporate entrepreneurship. Califormia Management Review, 26 (3) 154-166. doi: $10.2307 / 41165086$

Coduras, A., Guerrero, M. \& Peña, I. (2011). Emprendimiento corporativo en España. GEM España: Fundación Xavier de Salas para la Dirección General de Política de la Pyme.

Collins, O. \& Moore, D. G. (1970). The organization makers. New York: Appleton

Covin, J. G. \& Slevin, D. P. (1991). A Conceptual Model of Entrepreneurship as Firm Behavior. Entrepreneurship Theory and Practice, 16 (1), 7-25.

De Villiers-Scheepers, M. J. (2012). Antecedents of strategic corporate entrepreneurship. European Business Review, 24 (5), 400-424. doi: https://doi.org/10.1108/09555341211254508

Dess, G. G., Lumpkin, G. T. \& Covin, J. G. (1997). Entrepreneurial strategy making and firm performance: tests of contingency and configurational models. Strategic Management Journal, 18 (9), 677-695. doi: https://doi.org/10.1002/(SICI)10970266(199710)18:9<677::AIDSMJ905>3.0.CO;2-Q

Ellis, R. J. \& Taylor, N. T. (1987). Specifying entrepreneurship. En N. C. Churchill, J. A. Hornaday, B. A. Kirchhoff, O. J. Krasner, \& K. H. Vesper (Eds.). Frontiers of entrepreneurship research, pp. 527-541. Wellesley, MA: Babson College

Guth, W. D. \& Ginsberg, A. (1990). Guest Editors' Introduction: Corporate Entrepreneurship. Strategic Management Journal, 11, 5-15.

Hornsby, J. S., Kuratko, D. F. \& Zahra, S. A. (2002). Middle managers' perception of the internal environment for corporate entrepreneurship: assessing a measurement scale. Journal of Business Venturing, 17 (3), 253-273. doi: https:/ / doi.org/10.1016/S08839026(00)00059-8

Ireland, R. D., Covin, J. G. \& Kuratko D. F. (2009). Conceptualizing corporate entrepreneurship strategy. Entrepreneurship Theory and Practice, 33 (1), 19-46. doi: https://doi.org/10.1111/j.1540-6520.2008.00279.x

Jennings, D. F. \& Lumpkin, J. R. (1989). Functioning Modeling Corporate Entrepreneurship: An Empirical Integrative Analysis. Journal of Management, 15 (3), 485-503.

Jones, G. R. \& Butler, J. E. (1992). Managing internal corporate entrepreneurship: An agency theory perspective. Journal of Management. 18, 733-749. doi: https:// doi.org/10.1177/014920639201800408

Kearney, C., Hisrich, R. D. \& Roche, F. W. (2010). Change management through entrepreneurship in public sector enterprises. Journal of Developmental Entrepreneurship, 15 (4), 415-437. doi: https://doi.org/10.1142/S1084946710001646

Kearney, C., Hisrich, R. D. \& Antoncic, B. (2013). The mediating role of corporate entrepreneurship for external environment effects on performance. Journal of Business Economics and Management, 14 (1), 328-357. doi: https://doi.org/10.3846/16111699.2012.720592

Ketchen, D. J., Ireland, R. D. \& Snow, C. C. (2007). Strategic entrepreneurship, collaborative innovation, and wealth creation. Strategic Entrepreneurship Journal, 1 (3-4), 371-385. doi: https://doi.org/10.1002/sej.20

Knight, G.A. (1997). Cross-cultural reliability and validity of a scale to measure firm entrepreneurial orientation. Journal of Business Venturing, 12, 213-225.

Kuratko, D. F., Ireland, R. D., Covin, J. G. \& Hornsby, J. S. (2005). A model of middle level managers' entrepreneural behavior. Entrepreneurship Theory and Practice, 29 (6), 699716.

Kuratko, D. F. (2010). Corporate Entrepreneurship: An Introduction and Research Review. In Z. J. Acs \& D. B. Audretsch (Eds.). Handbook of Entrepreneurship Research, pp. 129-163. Springer New York. Recuperado de http://link.springer.com/chapter/10.1007/9781-441911919_6

Kuratko, D. F. \& Audretsch, D. B. (2013). Clarifying the domains of corporate entrepreneurship. International Entrepreneurship and Management Journal, 9 (3), 323-335. doi: https:// doi.org/10.1007/s11365-013-0257-4

Kuratko, D. F., Hornsby, J. S. \& Covin, J. G. (2014). Diagnosing a firm's internal environment for corporate entrepreneurship. Business Horizons, 57 (1), 37-47. doi: https://doi.org/10.1016/j.bushor.2013.08.009

Lumpkin, G. T. \& Dess, G. G. (1996). Clarifying the Entrepreneurial Orientation Construct and Linking It to Performance. The Academy of Management Review, 21 (1), 135-172.

Miles, M. B. \& Huberman, A. M. (1994). Qualitative Data Analysis: An expanded source book. United States: SAGE.

Miles, M. B., Huberman, A. M. \& Saldaña, J. (2013). Qualitative Data Analysis: A Methods Sourcebook (3rd Ed.). United States: SAGE. 
Miller, D. (1983). The Correlates of Entrepreneurship in Three Types of Firms. Management Science, 29 (7), 770-791. doi: https://doi.org/10.1287/mnsc.29.7.770

Morris, M. H., Kuratko, D. F., Covin, J. G. (2008). Corporate Entrepreneurship \& Innovation, Entrepreneurial Development within Organizations (2nd Ed.). United States: Thomson South-Western

Nelson, R. \& Winter, S. G. (1982) An evolutionary theory of economic change. Cambridge: Harvard University Press.

Nelson, R. \& Winter, S. G. (2002). Evolutionary theorizing in economics. Journal of Economic Perspectives, 16 (2), 23-46

Nielsen, R. P., Peters, M. P. \& Hisrich, R. D. (1985). Intrapreneurship strategy for internal markets: corporate, non-profit and government institution cases. Stategic Managemet Journal, 6 (2), 181-189. doi: https://doi.org/10.1002/smj.4250060207

Parker, S. C. (2011). Intrapreneurship or entrepreneurship? Journal of Business Venturing, 26 (1), 19-34. doi: https://doi.org/10.1016/j.jbusvent.2009.07.003

Pinchot, G. (1985). Intrapreneuring: Why You Don't Have to Leave the Corporation to Become an Entrepreneur. United States: Harper \& Row

Rutherford, M. W. \& Holt, D. T. (2007). Corporate entrepreneurship: An empirical look at the innovativeness dimension and its antecedents. Journal of Organizational Change Management, 20 (3), 429-446. doi: https://doi.org/10.1108/09534810710740227

Sakhari, K. (2016). Corporate Entrepreneurship: A Review and Future Research Agenda. Technology Innovation Management Review, 6 (8), 5-18.

Schindehutte, M., Morris, M. H., \& Kuratko, D. F. (2000). Triggering events, corporate entrepreneurship and the marketing function. Journal of Marketing Theory and Practice, 8 (2), 18-30.

Schollhammer, H. (1982). Internal corporate entrepreneurship. En C.A. Klent, D.L. Sexton y K.H. Vesper (Eds.). Encyclopedia of entrepreneurship, pp. 209-229. Englewood Cliffs, NJ: Prentice Hall.

Sharma, P. \& Chrisman, J. J. (1999). Toward a Reconciliation of the Definitional Issues in the Field of Corporate Entrepreneurship, Entrepreneurship Theory and Practice, 11-27.

Schumpeter, J. (1934). The Theory of Economic Development. Cambridge, MA: Harvard University Press.

Stevenson, H. H. \& Jarillo, J. C. (1990). A paradigm of entrepreneurship: Entrepreneurial management. Strategic Management Journal [Special issue], 11, 17-27.

Strauss, A. L. \& Corbin, J. M. (1990). Basics of qualitative research: grounded theory procedures and techniques. United States: SAGE.

Teece, D., \& Pisano, G. (1994). The dynamic capabilities of firms: an introduction. Industrial and Corporate Change, 3 (3), 537-556.

Teece, D. J., Pisano, G. \& Shuen, A. (1997). Dynamic capabilities and strategic management. Strategic Management Journal, 509-533.

Teece, D. J. \& Al-Aali, A. Y. (2013). Knowledge, Entrepreneurship, and Capabilities: Revising the Theory of the MNE. Universia Bussines Review, 4, 18-32.

Turró, A., Lopez, L. \& Urbano, D. (2013). Intrapreneurship conditioning factors from a resourcebased theory. European Journal International Management, 7 (3), 315-332.

Turró, A., Urbano, D. \& Peris-Ortiz, M. (2014). Culture and innovation: The moderating effect of cultural values on corporate entrepreneurship. Technological Forecasting and Social Change, 88, 360-369. doi: https://doi.org/10.1016/j.techfore.2013.10.004

Weeks, R. (2008). Nurturing a culture and climate of resilience to navigate the whitewaters of the South African dual economy. Journal of Contemporary Management, 5, 123-36.

Yoguel, G., Barletta, F. \& Pereira, M. (2013). De Schumpeter a los Postschumpeterianos: viejas y nuevas dimensiones analíticas. Revista Problemas del Desarrollo, 174 (44), 35-59.

Zahra, S. A. (1991). Predictors and financial outcomes of corporate entrepreneurship: An exploratory study. Journal of Business Venturing, 6 (4), 259-285. doi: https:/ / doi.org/10.1016/0883-9026(91)90019-A

Zahra, S. A. (1993). Environment, corporate entrepreneurship, and financial performance: a taxonomic approach. Journal of Business Venturing, 8 (4), 319-40. doi: https://doi.org/10.1016/0883-9026(93)90003-N

Zahra, S. A., Jennings, D. F. \& Kuratko, D. F. (1999). The Antecedents and Consequences of Firm-Level Entrepreneurship: The State of the Field. Entrepreneurship Theory and Practice, winter, 45. 


\section{NOTAS}

${ }^{i}$ Este artículo es resultado de la investigación titulada "Modelo de intraemprendimiento en México: factores causales y consecuentes", desarrollada en la Universidad Autónoma de Tamaulipas como parte del Programa de Doctorado en Ciencias Administrativas reconocido ante el Padrón Nacional de Posgrados de Calidad del Consejo Nacional de Ciencia y Tecnología. Cd. Victoria, Tamaulipas, http://www.uat.edu.mx/ Fecha de recepción (05/04/2018). Fecha de aceptación $(11 / 05 / 2018)$.

ii El interés surgido en tal periodo por esta nueva moda de gestión responde, por una parte, a los cambios contextuales derivados de la reestructuración de las condiciones económicas tras la culminación de la II Guerra Mundial, y por otra, al progreso de los sistemas de comunicaciones y transporte, los cuales propiciaron la modificación de las estructuras de mercado y con ello, el desarrollo de notables aportaciones teórico-empíricas en materia de gestión, innovación y estrategia (Chandler, 1997; Ghemawat, 2000, 2003; Peterson \& Berger, 1971).

iii En la literatura anglosajona se aborda el término "corporate venturing” para referirse a la “...persecución y la entrada en nuevos negocios dentro de la organización existente...” (Antoncic \& Hisrich, 2001: 495). Se han encontrado definiciones como "empresas riesgo", "capital riesgo" o "capital emprendedor" como traducciones aceptables al mismo.

\section{AUTORAS}

\section{EsTHELA GALVÁN VELA}

Magister en Comunicación Académica; Magister en Dirección Empresarial con Énfasis en Administración Estratégica; Doctorante en Ciencias Administrativas en la Universidad Autónoma de Tamaulipas, Ciudad Victoria, www.uat.edu.mx . Becaria del Consejo Nacional de Ciencia y Tecnología. Correo electrónico: gavela2012@gmail.com

MÓNICA L. SÁNCHEZ LIMÓN

Doctora en Ciencias de la Administración por la Universidad Nacional Autónoma de México. Miembro Nivel 1 del Sistema Nacional de Investigadores del Consejo Nacional de Ciencia y Tecnología. Profesor de Tiempo Completo de la Universidad Autónoma de Tamaulipas, Ciudad Victoria, www.uat.edu.mx. Correo electrónico: msanchel@gmail.com 